\title{
Towards understanding pre-mRNA splicing mechanisms and the role of SR proteins
}

\begin{abstract}
Alternative pre-mRNA splicing provides a source of vast protein diversity by removing noncoding sequences (introns) and accurately linking different exonic regions in the correct reading frame. The regulation of alternative splicing is essential for various cellular functions in both pathological and physiological conditions. In eukaryotic cells, this process is commonly used to increase proteomic diversity and to control gene expression either co- or post-transcriptionally. Alternative splicing occurs within a megadalton-sized, multicomponent machine consisting of RNA and proteins; during the splicing process, this complex undergoes dynamic changes via RNAÏ RNA, proteinï protein and RNAï protein interactions. Co-transcriptional splicing functionally integrates the transcriptional machinery, thereby enabling the two processes to influence one another, whereas post-transcriptional splicing facilitates the coupling of RNA splicing with post-splicing events. This review addresses the structural aspects of spliceosomes and the mechanistic implications of their stepwise assembly on the regulation of pre-mRNA splicing. Moreover, the role of phosphorylation-based, signal-induced changes in the regulation of the splicing process is demonstrated.
\end{abstract}

Keyword: Pre-mRNA splicing; Post-transcriptional splicing; Co-transcriptional splicing; Post-transcriptional splicing; SR proteins 